\title{
Numerical Model for the Determination of the Soil Retention Curve from Global Characteristics Obtained via a Centrifuge
}

\author{
B. Malengier, J. Kačur and P. Kišoň
}

\begin{abstract}
A novel centrifuge set-up for the study of unsaturated flow characteristics in porous media is examined. In this set-up, simple boundary conditions can be used, but a free moving boundary between unsaturated-saturated flow arises. A precise and numerically efficient approximation is presented for the mathematical model based on Richards' nonlinear and degenerate equation expressed in terms of effective saturation using the Van Genuchten-Mualem approach for the soil parameters in the unsaturated zone. Sensitivity of the measurable quantities (rotational moment, center of gravity and time period to achieve quasi steady state) on the soil parameters is investigated in several numerical experiments. They show that the setup is suitable for the determination of the soil parameters via the solution of an inverse problem in an iterative way.
\end{abstract}

\section{Introduction}

To predict the flow and solute transport in soils, one needs the soil hydraulic properties in terms of soil parameters. Once determined, these parameters can be used as input data in the governing mathematical model. For unsaturated flow, this model is given in terms of the saturation and the pressure head in Richards' equation (see below), which is a nonlinear and degenerate parabolic equation. Furthermore, when

\footnotetext{
B. Malengier

Department of Mathematical Analysis, Research Group $\mathrm{NaM}^{2}$, Ghent University, Galglaan 2, B9000 Ghent, Belgium, e-mail: bm@cage.UGent.be, Corresponding author

J. Kačur

Faculty of Mathematics, Physics and Informatics, Comenius University Bratislava, Slovakia, email: kacur@fmph.uniba.sk

P. Kišoň

Faculty of Mathematics, Physics and Informatics, Comenius University Bratislava, Slovakia, email: kisson@fmph.uniba.sk
} 
part of the sample is saturated, free boundaries between the saturated zone and the partially saturated zone arise, as well as between the dry and the partially saturated zone. This is a major problem for many modeling approaches, leading to experimental set-ups that avoid the formation of these boundaries.

The soil retention and hydraulic permeability functions linking the saturation and pressure head for unsaturated flow are expressed using the Van Genuchten-Mualem ansatz by means of soil parameters. Measuring these soil parameters is usually time consuming and tedious, especially for low conductive porous media. Several set-ups based on centrifugation have been proposed to obtain a large acceleration of the processes involved, see $[2,4,5,8,3]$ and citations therein. These techniques have several disadvantages. Aiming for a steady-state flow regime inside the centrifuge [2, 4] requires expensive and/or complex apparatus, and obtains only a few water content versus conductivity measurements per run. Also, transient set-ups based on keeping a top boundary at a fixed prescribed setting [3] are expensive. The quasi-steady centrifuge (QSC) method [1] is a simpler technique (a slowly emptying reservoir at the top that is refilled when needed), but requires that the criterion for steadiness of flow through the sample is relaxed, leading to higher uncertainty in the obtained results.

The alternatives for determining conductivity with a steady-state flow, combine transient flow with parameter estimation techniques, see e.g. [3, 8]. In this way, the conductivity and retention curve can be determined inversely over a large saturation domain. These methods require experiments of some state variables which relate to the conductivity. One-step or multi-step outflow methods are common in column experiments. The measurements are then used to estimate the hydraulic parameters. This technique is transferred to the centrifuge device in [8]. Good results are obtained, but there remain some disadvantages to this technique: there are few measurements close to saturation, leading to a high error in the prediction of the conductivity close to saturation, the sample needs to be disturbed to introduce electrodes, and there is a very long waiting time in order to achieve equilibrium when the equilibrium analysis approach is used.

The main goal of this manuscript is to develop a precise numerical method enabling to determine the soil parameters (via solution of inverse problem) in a very simple way requiring very cheap measurements.

In this Chapter we focus on a partially saturated sample which is sealed at the right boundary (from the center of centrifuge) and has no inflow at the left boundary. The only measurements required are the rotational momentum and the center of gravity of the sample at several time values, preferably also at the equilibria corresponding to predetermined rotational speeds. These measurements are sufficient due to the fact that the saturation profile at the equilibria do not depend on the initial distribution of water in the specimen, but only on its amount, which, when the right boundary of the sample is sealed, is identical in all equilibria.

To use this procedure, we have to face serious difficulties in the numerical modeling. The main one is that if the right side of the sample reaches effective saturation, an interface between partially saturated zone and saturated zone appears. This boundary is very difficult to control numerically, causing problems with the mass balance conservation which is very important in this set-up. 
To reach the equilibrium is an infinite asymptotic process, but after some time (e.g. 1-3 days for low conductive material) the change of the rotational momentum and of the center of gravity can no longer be measured. At that moment, the rotational speed is increased, and the system moves towards a new corresponding equilibrium. Note that even when equilibrium was not reached and a small error is present in the measurements of the rotational momentum and the center of gravity, this will not influence the error at the higher equilibrium level. This error depends only on the running time of centrifugation at the actual rotational speed. The differences between applied rotational speeds are chosen in such a way that that the differences in outputs (rotational momentum and center of gravity) are technically well distinguishable.

Next, the soil parameters and eventually the amount of originally infiltrated water, can be determined by minimizing a cost functional expressing the distance between the measured and the computed output, e.g., with the Levenberg-Marquard method. The advantage of this approach is that the full range of saturation values are present in the setup, while preventing outflow means equilibrium can be obtained faster. However, due to the set-up, it is clear that the water flows from the unsaturated zone to the saturated zone, with no flow occurring in the saturated zone. Indeed, we notice that the rotational momentum and center of gravity are not sufficiently sensitive on the "saturated hydraulic conductivity". This parameter is hence better determined from saturated flow experiments, see eg [5].

In the numerical method, we reduce the mathematical model to a system of ordinary differential equations (ODE) using the method of lines (MOL), which has already been successfully applied to Richards' equation in e.g. [6]. As a variation, a reduction to a system of ODE and algebraic equations (DAE) is considered. Our main contribution is in correctly handling the moving free boundary. The obtained system can be solved with ODE/DAE solvers for stiff systems.. The numerical method can be successfully applied in other centrifugation settings (concerning control of the inflow, or control of the outflow) as, e.g., in $[8,3]$.

In Section 2, we present the mathematical model, giving specific attention to the movement of the free boundary. In Section 3 the numerical method based on the MOL approach is given, while in Section 4 the approach to determine the saturated hydraulic conductivity is explained. We finish in Section 5 with several numerical experiments showing the sensitivity of the output parameters on the soil parameters.

\section{Mathematical Model}

We consider a one dimensional model for a partially saturated sample in the form of a tube. The tube starts (top or left boundary) at the distance $r=r_{0}$ from the center of the centrifuge and ends at the distance $r=r_{0}+L$. The right boundary of the specimen is isolated. Flow in porous media under centrifugation is modeled by Darcy's equation in the saturated region and by Richards' equation in the unsaturated region (see, e.g., [8],[3]). So 


$$
\partial_{r}\left[K_{s}\left(\partial_{r} h-\frac{\omega^{2}}{g} r\right)\right]=0
$$

in the saturated region, and

$$
\partial_{t} \theta=\partial_{r}\left[k(\theta)\left(\partial_{r} h-\frac{\omega^{2}}{g} r\right)\right],
$$

in the unsaturated region. Here, $h$ is the piesometric head, $\theta$ the saturation of the porous medium, $\omega$ the angular speed of rotation (in radians per second), $K_{s}$ the hydraulic conductivity in the saturated region, $g$ the gravitational constant and the function $k(\theta)$ describes the hydraulic conductivity in the unsaturated region. Denote by $u=\frac{\theta-\theta_{r}}{\theta_{s}-\theta_{r}}$ the effective saturation, where $\theta_{s}$ is the volumetric water content at saturation and $\theta_{r}$ is the residual volumetric water content. We have $u \in(0,1)$, since $\theta \in\left(\theta_{s}, \theta_{r}\right)$. The soil hydraulic properties are represented by empirical expressions (see [7]),

$$
u=\frac{1}{\left(1+(\gamma h)^{n}\right)^{m}}, \quad h \in(-\infty, 0), \quad k(u)=K_{s} u^{1 / 2}\left[1-\left(1-u^{1 / m}\right)^{m}\right]^{2},
$$

where $m=1-1 / n, n>1$ and $\gamma$ are empirical soil parameters. Determining $\gamma, n$ and $K_{s}$ from the experiments means the soil retention curve has been determined. Note that we do not take hysteresis of the retention curve in consideration with this model.

It is possible to rewrite the flow in unsaturated form as

$$
\partial_{t} u=\partial_{r}\left(D(u) \partial_{r} u-\frac{\omega^{2}}{g} k(u) r\right)
$$

where

$$
D(u)=-\frac{K_{s}}{(n-1) \gamma\left(\theta_{s}-\theta_{r}\right)} u^{1 / 2-1 / m}\left(1-u^{1 / m}\right)^{-m} \times\left[1-\left(1-u^{1 / m}\right)^{m}\right]^{2} .
$$

Equation (4) is strongly nonlinear and degenerate. We note that $D(0)=0, D(1)=\infty$. Equilibria at the high rotational speed can be expected to have a fully saturated zone (supposing the initial amount of infiltrated water is sufficiently large), which appears at the right boundary and of which the front evolves to the left of the specimen (under non-decreasing rotational speed). We denote the position of this interface by $s(t)$. This saturated zone is governed by Darcy's equation, but $s(t)$ is unknown and time dependent. The time evolution of $s(t)$ is difficult to compute. The dynamics of this region is linked with the (finite) interface flux $q_{i}$

$$
q_{i}=-\left.\left(D(u) \partial_{r} u-\frac{\omega^{2}}{g} k(u) r\right)\right|_{r=s(t)},
$$


and based on a mass balance argument we can expect $\dot{s}(t)=-q_{i}$. Unfortunately, we cannot use this model for the determination of the time evolution of $s(t)$, since at $r=s(t)$ it holds $u=1$ and $D(1)=\infty$. Consequently, $\left.\partial_{r} u\right|_{r=s(t)}=0$.

If we transform Richards' equation in terms of the piesometric head using (3), we obtain

$$
d_{s}(h) \partial_{t} h=k_{0} \partial_{r}\left[\bar{k}(h) \partial_{r} h-\frac{\omega^{2}}{g} \bar{k}(h) r\right]
$$

with $k_{0}=\frac{K_{s}}{\theta_{s}-\theta_{r}}$, where $k_{0} \bar{k}(h)$ is the hydraulic conductivity function,

$$
\bar{k}(h)=\frac{1}{\left(1+(\gamma h)^{n}\right)^{m / 2}}\left(1-\frac{(\gamma h)^{n-1}}{\left(1+(\gamma h)^{n}\right)^{m}}\right)^{2},
$$

and the specific moisture capacity function $d_{s}(h)=\mathrm{d} u / \mathrm{d} h$ is given by

$$
d_{S}(h)=-\gamma(n-1) \frac{(\gamma h)^{n-1}}{\left(1+(\gamma h)^{n}\right)^{1+m}} .
$$

We can see that $\bar{k}(h) \rightarrow 1$ for $h \rightarrow 0$. In Fig. 1 we present the graph of the functions $\bar{k}(h)$ and $100 d_{s}(h)$ for $h \in(-200,0)$, and parameter values $K_{s}=2.410^{-5}, n=2.81$, $\gamma=-0.0189$. As we can see, Eq. (6) also degenerates at $h=0$. This has to be taken

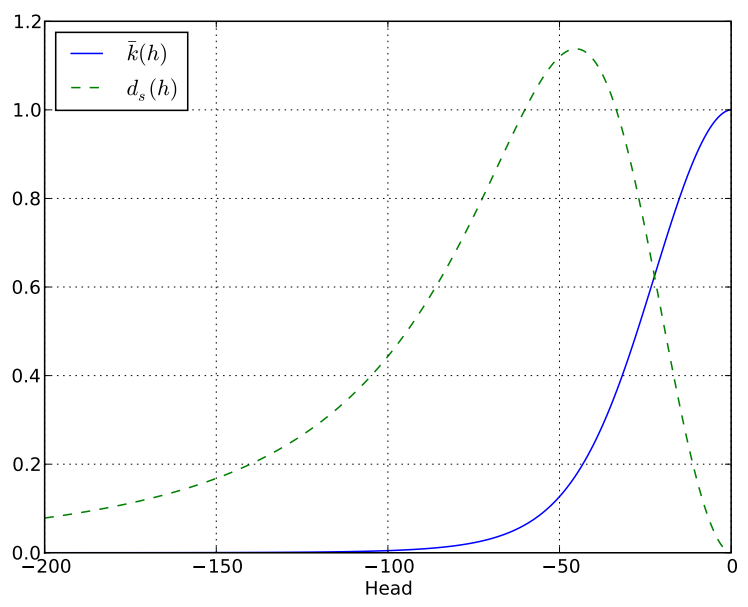

Figure $1 \bar{k}(h)$ and $100 \times d_{s}(h)$ for $n=2.81, \gamma=-0.0189$

into account when saturation becomes 1 at the right boundary of specimen. After this moment, $t=t_{1}$, the mathematical model must be changed to reflect the physical phenomenon. At the right hand side of the (isolated) specimen appears a saturated zone with an interface $s(t)$ moving from the right boundary to the left. The flux at 
the interface $s(t)$ is equal to $-\dot{s}(t)$, but also in this pressure-head form of Richards' equation it is difficult to approximate correctly $\left.\partial_{t} h\right|_{x=s(t)}$, which leads to a significant error in the mass balance.

Therefore, to determine the interface $s(t)$, we will consider the algebraic equation

$$
\int_{r_{0}}^{r_{0}+L} u(h(x, t)) \mathrm{d} t+L-s(t)=M_{w}, \quad s(0)=L,
$$

where $M_{w}$ is the amount of infiltrated water (which remains constant during the centrifugation). This condition reflects the global mass balance in the specimen and does not suffer from a flux approximation at $r=r_{0}+s(t)$.

Then, mathematical model (6) only needs to be solved over the interval $r \in$ $\left(r_{0}, r_{0}+s(t)\right)$ with right boundary condition $h\left(r_{0}+s(t)\right)=0$ for all $t$. We approximate this mathematical model in the next section.

\section{Numerical Method}

For the output parameters that will be measured (gravity center and rotational momentum), there is no need to model the head in the saturated zone, as we consider the compressibility of water to be negligible. The numerical approximation of (6)-(7) results in a coupled system of a partial differential equation (PDE) and an algebraic equation. Moreover, the solution domain is a moving region, with unknown interface $s(t)$, which has to be determined.

We shift (6) to the domain $r \in(0, s(t))$ and use the fixed domain transformation $y=\frac{r}{s(t)}$. This gives

$$
d_{s}(h)\left(\mathrm{d}_{t} h(y, t)-y \frac{\dot{s}(t)}{s(t)} \partial_{y} h\right)=k_{0} \frac{1}{s(t)^{2}} \partial_{y}\left(\bar{k}(h) \partial_{y} h-\bar{k}(h) \frac{\omega^{2} s}{g}\left(r_{0}+y s(t)\right)\right) .
$$

Consider the space discretization $0=y_{0}<y_{1}<\ldots<y_{i}<\ldots<y_{N}=1$, and $\alpha_{0}=$ $0, \alpha_{i}:=y_{i}-y_{i-1}, i=1, \ldots, N$ and integrate $(8)$ over $I_{i}:=\left(y_{i-1 / 2}, y_{i+1 / 2}\right)$ for $i=$ $1, \ldots, N-1$ where $y_{i-1 / 2}:=\left(y_{i}+y_{i-1}\right) / 2, y_{i+1 / 2}:=\left(y_{i}+y_{i+1}\right) / 2$.

We denote by $h_{i}(t) \approx h\left(y_{i}, t\right), \forall i=1, \ldots, N-1$, and approximate $\mathrm{d}_{t} h(y, t) \approx \dot{h}_{i}(t)$ in the interval $I_{i}$. We approximate

$$
\partial_{y} h_{y=y_{i+1 / 2}} \approx \frac{h_{i+1}(t)-h_{i}(t)}{\alpha_{i+1}}=: \partial^{+} h_{i}
$$

and similarly we approximate $\left.\partial_{y} h\right|_{y=y_{i-1 / 2}}$ and denote it by : $\partial^{-} h_{i}$. Let $\mathscr{L}\left(z ; y_{i}\right)$ be the second order Lagrange polynomial crossing the points $\left(y_{i-1}, h_{i-1}\right),\left(y_{i}, h_{i}\right)$ and $\left(y_{i+1}, h_{i+1}\right)$. We use the abbreviation $k_{i+1 / 2}:=\bar{k}\left(h_{y_{i+1 / 2}}\right)$. Then, the approximation of (8) (based on finite volume type approximation) at the point $y=y_{i}$ reads as follows 


$$
\begin{gathered}
d_{s}\left(h_{i}\right)\left(\dot{h}_{i}-\left.\frac{\dot{s} y_{i}}{s} \frac{d \mathscr{L}\left(z ; y_{i}\right)}{d z}\right|_{z=y_{i}}\right)=k_{0} \frac{2}{\alpha_{i}+\alpha_{i+1}} \frac{1}{s^{2}}\left[k_{i+1 / 2} \partial^{+} h_{i}-k_{i-1 / 2} \partial^{-} h_{i}-\right. \\
\left.\frac{\omega^{2} s}{g}\left(k_{i+1 / 2}\left(r_{0}+s y_{i+1 / 2}\right)-k_{i-1 / 2}\left(r_{0}+s y_{i-1 / 2}\right)\right)\right]
\end{gathered}
$$

for $i=1, \ldots, N-1$. We add the corresponding equation at point $y_{0}$ taking into account that the flux is zero there. In a similar way as in (9) (following the finite volume type of approximation) we obtain

$$
d_{s}\left(h_{0}\right) \dot{h}_{0}=k_{0} \frac{2}{\alpha(1)} \frac{1}{s^{2}} \times\left[k_{1 / 2} \partial^{+} h_{1}-\frac{\omega^{2} s}{g}\left(k_{1 / 2}\left(r_{0}+s y_{1 / 2}\right)\right)\right] .
$$

At the point $y_{N}=1$ we have $h_{N}(t)=0$, so no additional equation is considered. We approximate the amount of water $M_{w}$ using the trapezoidal rule for the integration. Define

$$
Q(t) \approx u_{0} \alpha_{1} / 2+\alpha_{N} / 2+\sum_{1}^{N-1} \frac{\alpha_{i}+\alpha_{i+1}}{2} u_{i}
$$

where $u_{i}=\frac{1}{\left(1+\left(\gamma h_{i}\right)^{n}\right)^{m}}$. Then, system (9)-(10) will be completed by the algebraic equation

$$
0=L-s(t)[1-Q(t)]-M_{w} .
$$

This algebraic equation is used instead of an ODE equation that models $\dot{s}(t)$. System (9)-(11) is degenerate and is of the form

$$
M(t, z) \dot{z}(t)=f(t, z)
$$

where $z=\left(h_{0}, h_{1}, \ldots, h_{N-1}, s\right)$. The last equation of this system is just (11). This system can be readily solved, e.g., by the solver "ode15s" in MATLAB ${ }^{\circledR}$ or the "ida" solver of the Sundials package.

As is usual with these solvers, some regularization in (11) is needed as well as a tuning of the space discretization. Most important is to have a "good" starting point.

If the equilibria have the property $h_{N}<0$, then no interface appears. It is then needed to set $s(t):=L$ in the previous mathematical model and replace algebraic equation (11) by an ODE equation for $\dot{h}_{N}$ which will be similar to (10). Successively increasing the rotational speed of the centrifuge increases the head at the right boundary. The model remains in the state where $s(t):=L$ up to the point when $h(N)=0$, at which point the computation is automatically halted. The full model (9)-(11) is used onwards to compute the equilibrium states.

In numerical equilibrium experiments it is observed, as expected, that the values of the rotational moment $M_{r}$ and the center of gravity $G_{c}$ are not very sensitive to the $K_{s}$ parameter. Also, the transient experiments where the time sections between different equilibria are measured, are not very sensitive. The saturated conductivity $K_{s}$ can only be determined from measurements of $M_{r}, G_{c}$ that are accurate up to 3 digits. Therefore, another method must be used for the determination of $K_{s}$. 


\section{Alternative Experiments}

\subsection{Saturated Flow}

For the determination of the saturated conductivity, we propose to use the method put forward in [5], a water reservoir put to the left of a saturated sample and collection of the water in an outflow reservoir, with the addition of allowing for transient measurements. We specifically use the ability to measure when a reservoir has completely drained out, combined with the measurements of the rotational moment.

This leads to the following equation for the dropping water level $\ell(t)$ in the reservoir,

$$
\left.\dot{\ell}(t)=-K_{s} \frac{\omega^{2}}{2 g L}\left[L^{2}-\ell(t)^{2}+2 r_{0}(L+\ell(t))\right)\right] \equiv-q_{F}(t),
$$

with $\ell(0)=l_{0}$ and $\ell\left(T_{e}\right)=0$. Solving this ODE, we obtain the relation between $T_{e}$ and $K_{s}$, whereas $\ell(t)$ fully determines the change of the rotational moment $M_{r}(t)$ over time.

\subsection{Water Reservoir and Outflow Reservoir}

The mathematical and numerical model presented can be extended also to allow for a water reservoir to the left of an unsaturated sample, and an outflow reservoir to the right. This allows several different centrifugation experiments to be performed, and allows to change the set-up during an experiment. For example, the following scenario is possible: 1 . Start from a saturated sample and a water reservoir to the left. This makes it possible to determine $K_{s}$. 2. Continue with outflow of the water content, making the sample unsaturated. 3. Isolate the right boundary (that is, close it), which means we have the problem as described in the previous two Sections. 4. Continue step 2 and 3 of above so as to change the global water content.

The advantage of the above centrifugation scenario is that all parameters can be determined with one ground sample, and that more saturation levels are sampled during the entire experiment. The main point to arrive at an accurate solution of the model doesn't change: an algebraic equation for mass balance determines the difficulty to control unknowns. With a closed right boundary, this is the moving interface, with an open boundary, this will be the outflow flux.

\section{Numerical Experiments}

For the first experiments we use as data $r_{0}=10, L=10, \omega=30, K_{s}=2.410^{-5}$, $\theta_{r}=0.02, \theta_{s}=0.4, \gamma=-0.0189, n=2.81$, except where sequences are compared 
to investigate the sensitivity of the set-up on the parameters. A uniformly distributed space discretization with $N=40$ grid points is used.

The formulas for $M_{r}, G_{c}$ and $M_{w}$ at time $t$ are:

$$
\begin{gathered}
M_{r}=\frac{s(t)}{2} \int_{0}^{1}\left(r_{0}+s(t) z\right)^{2} u(t, z) d z+\frac{1}{6}\left(L^{3}-s(t)^{3}\right), \\
M_{w}=s(t) \int_{0}^{1} u(t, z) d z+\frac{1}{2}\left(L^{2}-s(t)^{2}\right), \quad G_{c}=s(t) \int_{0}^{1} y u(t, z) d z / M_{w},
\end{gathered}
$$

and are all evaluated numerically using the trapezoidal rule. Note that if $u(t, 1)<1$ then $s(t)=L$. The sensitivity of the measured quantities on the changing water content is very good. The following experiments allow to determine the contribution a change in the different soil parameters has on the measurements.

\subsection{Reaching Equilibrium}

To investigate the head profiles we start this experiment from the equilibrium corresponding to $\omega=40$ and a rotational speed $\omega=50$. The centrifuge normally operates up to $T_{e}=1.540 .000$ seconds. At that time, equilibrium for $\omega=50$ is almost reached. We compare 13 values, the starting value, 9 increasing time steps (with $\left.\Delta t_{j}=t_{j+1}-t_{j}=20002^{j}, j=1, \ldots, 9\right)$, the sensible end time step $T_{e}=770 \times 2.000 \mathrm{~s}$, and 2 extra time steps to investigate the very long time behavior. The measured val-

Table 1 Rotational momentum, center of gravity, water amount for Exp. 5.1

\begin{tabular}{|l|l|l|l|}
\hline$\frac{\mathrm{time}}{2000} \mathrm{~s}$ & $M_{r, e} 10^{-6}$ & $G_{c}$ & $M_{w}$ \\
\hline 0 & 1.5201 & 7.2512 & 4.0141 \\
\hline 1 & 1.5248 & 7.2813 & 4.0128 \\
\hline 3 & 1.5299 & 7.3119 & 4.0131 \\
\hline 7 & 1.5345 & 7.3413 & 4.0133 \\
\hline 15 & 1.5389 & 7.3697 & 4.0134 \\
\hline 31 & 1.5430 & 7.3972 & 4.0134 \\
\hline 63 & 1.5469 & 7.4234 & 4.0135 \\
\hline 127 & 1.5505 & 7.4478 & 4.0135 \\
\hline 255 & 1.5537 & 7.4699 & 4.0136 \\
\hline 511 & 1.5565 & 7.4893 & 4.0136 \\
\hline 770 & 1.5588 & 7.5056 & 4.0136 \\
\hline 1800 & 1.5645 & 7.5462 & 4.0133 \\
\hline 2300 & 1.5649 & 7.5494 & 4.0133 \\
\hline
\end{tabular}

ues for the rotational momentum, gravity center, and water amount, are given in Table 1. The small change between the last two values in Table 1 demonstrates that equilibrium is eventually reached. 
We can conclude that reaching equilibrium is a very slow process. The reason for this is that the hydraulic permeability at low head is negligibly small, so it takes a very long time to reach the equilibrium. If the centrifugation is continued, also the section with low head obtains the required parabolic shape associated with the equilibrium. Note however, that the other part of the head profile (for higher head values) is changing insignificantly. Therefore, we arrive at the conclusion that it makes sense to increase the rotational speed and not wait for these lower head values to stabilize.

\subsection{Dependence on $n$}

In this experiment, we demonstrate the sensitivity of $M_{r}$ and $G_{c}$ to the model parameter $n$. We start with a constant saturation $u=0.4$ and apply the rotational speed $\omega=20$. The centrifuge is operated for 800.000s. In Fig. 2 the obtained equilibrium profiles are depicted for successively $n=1.51 ; 1.81 ; 2.11 ; 2.41 ; 2.71 ; 2.81 ; 3.01$ and 3.31 .

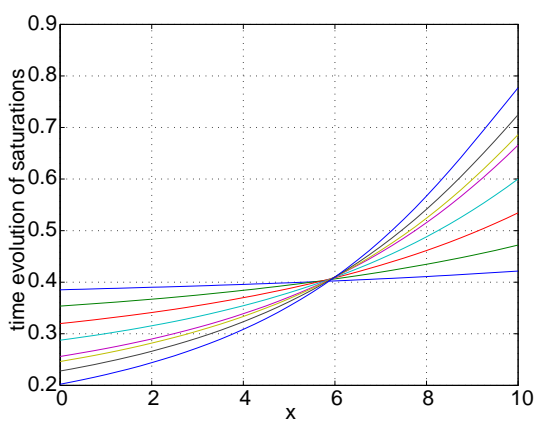

(a) Saturation profiles

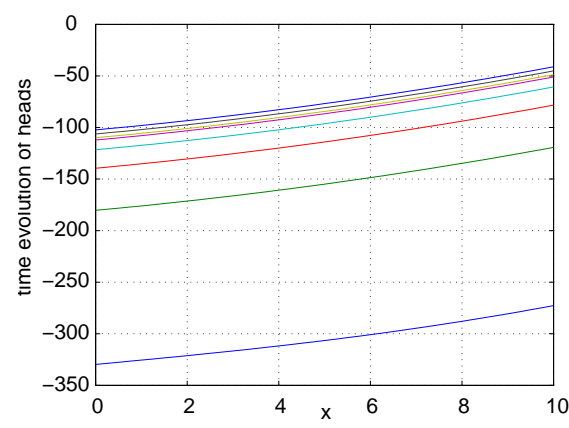

(b) Head profiles

Figure 2 Equilibrium profiles at $\omega=20$ for $n=1.51 ; 1.81 ; 2.11 ; 2.41 ; 2.71 ; 2.81 ; 3.01$ and 3.31 .

The resulting values for $M_{r}, G_{c}$ and $M_{w}$ are given in Table 2, and indicate a good sensitivity.

\subsection{Dependence on $\gamma$}

We now investigate the sensitivity of $M_{r}$ and $G_{c}$ to the $\gamma$ soil retention curve parameter. We again use a rotational speed of $\omega=50$, starting from the equilibrium position at $\omega=35$. As values for $\gamma$ we consider $\gamma=-\gamma_{0} 10^{2}$ with $\gamma_{0} \in(1.59 ; 2.19)$ 
Table 2 Rotational momentum, center of gravity and water amount for Exp. 5.2

\begin{tabular}{|l|l|l|l|}
\hline$n$ & $M_{r} e .10^{-6}$ & $G_{c}$ & $M_{w}$ \\
\hline 1.51 & 0.1887 & 5.0736 & 4.0043 \\
\hline 1.81 & 0.1927 & 5.2391 & 4.0052 \\
\hline 2.11 & 0.2020 & 5.6188 & 4.0043 \\
\hline 2.41 & 0.2068 & 5.8096 & 4.0062 \\
\hline 2.71 & 0.2083 & 5.8701 & 4.0054 \\
\hline 2.81 & 0.2112 & 5.9870 & 4.052 \\
\hline 3.01 & 0.2153 & 6.1524 & 4.0060 \\
\hline 3.31 & 1.5505 & 7.4478 & 4.0135 \\
\hline
\end{tabular}

where increments of size 0.1 are used. The values of $M_{r}$ and $G_{c}$ are listed in Table 3 and Table 4, respectively. The corresponding saturation and head profiles at time section $t=10^{5}$ are given in Fig. 3. In Tables 3 and 4 the water amount is 4.05. The

Table 3 Rotational momentum $M_{r} 10^{-6}$ for Exp. 5.3,

\begin{tabular}{|l|l|l|l|l|l|l|l|}
\hline time $\backslash \gamma_{0}$ & 1.59 & 1.69 & 1.79 & 1.89 & 1.99 & 2.09 & 2.19 \\
\hline \hline 1000 & 1.5189 & 1.5093 & 1.5013 & 1.4949 & 1.4896 & 1.4854 & 1.4819 \\
\hline 3000 & 1.5352 & 1.5213 & 1.5097 & 1.5000 & 1.4919 & 1.4852 & 1.4797 \\
\hline 5000 & 1.5438 & 1.5278 & 1.5144 & 1.5030 & 1.4935 & 1.4855 & 1.4788 \\
\hline $10^{4}$ & 1.5565 & 1.5376 & 1.5216 & 1.5079 & 1.4963 & 1.4864 & 1.4780 \\
\hline $5.10^{4}$ & 1.5901 & 1.5645 & 1.5423 & 1.5231 & 1.5063 & 1.4917 & 1.4791 \\
\hline $10^{5}$ & 1.6058 & 1.5777 & 1.5530 & 1.5313 & 1.5124 & 1.4958 & 1.4812 \\
\hline
\end{tabular}

Table 4 Center of gravity for Exp. 5.3

\begin{tabular}{|l|l|l|l|l|l|l|l|}
\hline time $\backslash \gamma_{0}$ & 1.59 & 1.69 & 1.79 & 1.89 & 1.99 & 2.09 & 2.19 \\
\hline \hline 1000 & 7.1117 & 7.1031 & 7.0948 & 7.0872 & 7.0805 & 7.0744 & 7.0693 \\
\hline 3000 & 7.1487 & 7.1379 & 7.1271 & 7.1170 & 7.1076 & 7.0990 & 7.0912 \\
\hline 5000 & 7.1697 & 7.1581 & 7.1464 & 7.1351 & 7.1245 & 7.1146 & 7.1056 \\
\hline $10^{4}$ & 7.2026 & 7.1903 & 7.1775 & 7.1649 & 7.1527 & 7.1411 & 7.1303 \\
\hline $5.10^{4}$ & 7.3012 & 7.2898 & 7.2763 & 7.2617 & 7.2466 & 7.2314 & 7.2167 \\
\hline $10^{5}$ & 7.3512 & 7.3429 & 7.3309 & 7.3167 & 7.3012 & 7.2851 & 7.2689 \\
\hline
\end{tabular}

sensitivity on $\gamma$ is less than that of $n$, but is sufficient. Nevertheless, taking transient information into account, as given in the rows of Tables 3 and 4, will benefit the determination of $\gamma$ via this experimental set-up. 


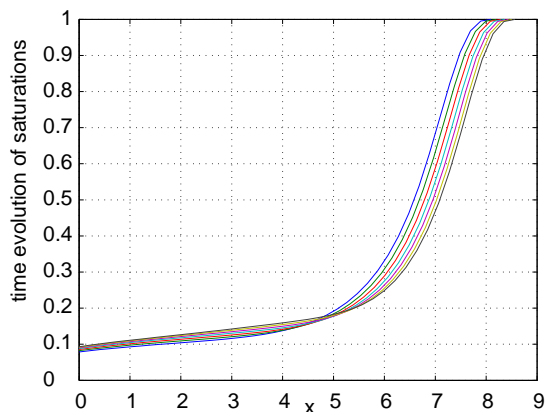

(a) Saturation profiles

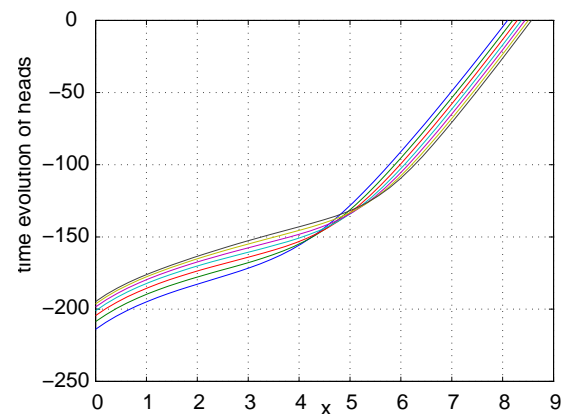

(b) Head profiles

Figure 3 Saturation profiles at equilibrium for $\omega=50, \quad \gamma_{0}=$ $1.59 ; 1.69 ; 1.79 ; 1.89 ; 1.99 ; 2.09 ; 2.19$

\subsection{Inverse determination of $\gamma, n$ and $K_{s}$}

In this numerical experiment, we use $r_{0}=30 L=10, \omega=20, K_{s}=2.4 .10^{-5}, \theta_{r}=$ $0.02, \theta_{s}=0.4, \gamma=-0.0189$ and $n=2.81$. The space discretization for $T \in\left(0, T_{1}\right)$ is not equidistant. Here $T_{1}$ is the time needed to empty the left water reservoir. We shall consider $N=40$ grid points with geometrical distribution as follows. The first space interval is $d_{1}=1 / 20$ and then $d_{i+1}=q d_{i}$ with $q<1$. Once the water reservoir is empty, a uniform space discretization with $N=40$ is used.

In this experiment, we restore the soil parameters applying the following centrifugation scenario. First, we centrifugate the fully saturated sample along the time $10^{4}$ and collect dat $a_{1}=\left\{M_{1}, G_{1}, M_{w, 1}\right\}$. Then, we isolate the right boundary of the sample and centrifugate it tor $t=5000$ s with rotational speed $\omega=15$. Then, we obtain data $_{2}=\left\{M_{2}, G_{2}, M_{w, 2}\right\}$ (where $M_{w, 2}=M_{w, 1}$, since we have zero output). After this, we repeat these two steps with the same sample at the same running time $t=5000 s$ and $\omega=20$. Successively we obtain data $_{3}=\left\{M_{3}, G_{3}, M_{w, 3}\right\}$, data $a_{4}=$ $\left\{M_{4}, G_{4}, M_{w, 4}\right\}$ where $\left(M_{w, 4}=M_{w, 3}\right)$ and continue up to data . Then, the total measurement data is represented by the vector data $=\left\{\right.$ data $_{1}$, data $_{2}, \ldots$, data $\}$. To imitate a realistic situation, we perturb every component of data by 0.01 .(rand -0.5 ), where rand is a generator of random numbers from $(0,1)$. This corresponds to $0.5 \%$ noise. Next, we apply the Levenberg-Marquardt method to restore the soil parameters, starting from initial parameters $\gamma=-0.01, n=2 ., K_{s}=1.610^{-5}$. The corresponding iterations of the LM method are presented in Table 5. 


\begin{tabular}{|l|l|l|l|l|}
\hline iteration & $-100 . \gamma$ & $n$ & $K_{S} 10^{5}$ & $R M S$ \\
\hline 0 & 1. & 2. & 1.6 & 3.3977 \\
\hline 1 & 1.7643 & 3.4612 & 1.6149 & $5.05710^{-2}$ \\
\hline 3 & 1.8555 & 2.8423 & 2.2320 & $2.87810^{-4}$ \\
\hline 5 & 1.8469 & 2.8519 & 2.2185 & $1.50610^{-4}$ \\
\hline
\end{tabular}

Table $5 \mathrm{LM}$-iterations for determination of $\gamma, n, K_{s}$

\section{Conclusion}

In this Chapter, it is shown that global characteristics measured with a centrifuge can be used to determine the soil retention curve of ground samples. In order for this to work, transient data must be used, different centrifugation scenario's must be coupled to obtain sufficient information, and a very precise numerical model must be used. Specifically, this model must be able to accurately track the moving interface. We further draw attention that in the alternative scenario using outflow, no outflow boundary condition is imposed. This gives more freedom to the experimentator. Instead, for all simulations, an algebraic equation based on mass balance is used to obtain a solution.

Acknowledgements The second and third author confirm financial support by the Slovak Research and Development Agency under contract APVV-0184-10 and VEGA-1/0502/09.

\section{References}

1. M.C. Caputo and J.R Nimmo. Quasi-steady centrifuge method for unsaturated hydraulic properties. Water Resour. Res., 41:W11504, 2005.

2. J.L. Conca and J.V. Wright. The ufa method for rapid, direct measurements of unsaturated transport properties in soil, sediment and rock. Aust. J. Soil Res., 36:291-315, 1998.

3. D. Constales and J. Kačur. Determination of soil parameters via the solution of inverse problems in infiltration. Computational Geosciences, 5:25-46, 2004.

4. R.J. Nimmo, K.C. Akstin, and K.A. Mello. Improved apparatus for measuring hydraulic conductivity at low water content. Soil Sci. Soc. Am. J., 56:1758-1761, 1992.

5. R.J. Nimmo and K.A. Mello. Centrifugal techniques for measuring saturated hydraulic conductivity. Water Resour. Res., 27(6):1263-1269, 1991.

6. M.D. Tocci and C.T. Kelley. Accurate and economical solution of the pressure-head form of richards' equation by the method of lines. Advances in Water Resources, 20(1):1-14, 1997.

7. M. Th. van Genuchten. A closed-form equation for predicting the hydraulic conductivity of unsaturated soils. Soil Sci.Soc.Am. J., 44:892-898, 1980.

8. J. Šimůnek and J.R. Nimmo. Estimating soil hydraulic parameters from transient flow experiments in a centrifuge using parameter optimizetion technique. Water Resour. Res., 41, 2005. 